\title{
Analysis of Jamming-deleted Self-normalized Cascade Diversity Combining Receiver Technology in MFSK/FFH
}

\author{
Junshi XIE ${ }^{1, a}$, Heng WANG ${ }^{1, b}$ \\ ${ }^{1}$ Nanjing Telecommunication Technology Research Institute of CESEC, Nanjing, 210007, China \\ aemail: xsjxsj_520@163.com, bemail:wangheng0987654321@126.com
}

Keywords: Jamming-deleted Self-normalized, Cascade Diversity Combining Receiver Technology.

\begin{abstract}
In the fast frequency hopping system(FFH), the diversity combining technology can effectively against the partial band jamming(PBJ), this paper studies an improved diversity combining technology, it is the jamming-deleted self-normalized diversity combining technology(JD-SNZ), The technology is based on interference intensity recognition, deleted interference diversity technology jump sample, the cascade technology for each hop envelope samples two weighted processing, make full use of the fast frequency hopping frequency diversity characteristics, improve diversity gain and enhance the system anti-interference ability. Simulation and analysis are carried out under the partial band Gauss noise jamming environment, and the results show that the proposed technology improves the ability of the system to resist partial band interference compared with the single diversity combining technology, and it is easier to implement on hardware.
\end{abstract}

\section{Introduction}

The frequency hopping has the characteristic of strong anti-interference, and is widely used in communication anti-jamming system. When the frequency hopping rate is high, it is difficult to implement the tracking jamming, and the effective jamming to the frequency hopping system is partial band jamming. To study the effect of eliminating or weakening the interference on the performance of the system is receiving widespread attention. In the fast frequency hopping system, if one hop information is repeated several times to send, the receiver acquires information from the demodulated signal of these repeated uncorrelated interference, and select or merge the process as a way of diversity, the diversity will reduce the partial band jamming effect on system.

Now FFH diversity combining have recently analyzed in references [1]-[5] .In the traditional technology, hard decision majority vote(HDMV),product (PCR),self-normalized(SNZ)diversity combining receiver was shown in [1]-[3] that are less simple and counter. While some technology use side information to acquire better performance, but the realization of this technology complicatedly, include maximum-likelihood technology(ML)[4].If two different diversity combining technology cascade, it can be stronger against PBJ than only one diversity technology.

\section{Jamming-deleted Self-normalized Diversity Combining Receiver Technology}

The implementation difficulty of time frequency diversity technique is the combination of diversity signals. In the fast frequency hopping system, we propose FFT group branch receive processing based on interference intensity, automatic identification, "adaptive interference jump" sample deletion of the variable dimension diversity technology based on the technology is a self normalization technology based on cascade technology, the hard decision of large numbers, since the election and the performance of the technology set equal normalization technology superior to the traditional, although slightly lower than the maximum likelihood diversity technology, but the technology hardware implementation, does not need to estimate the noise variance. Figure 1. Block diagram of frequency hopping and deletion self normalized diversity combining technology. 


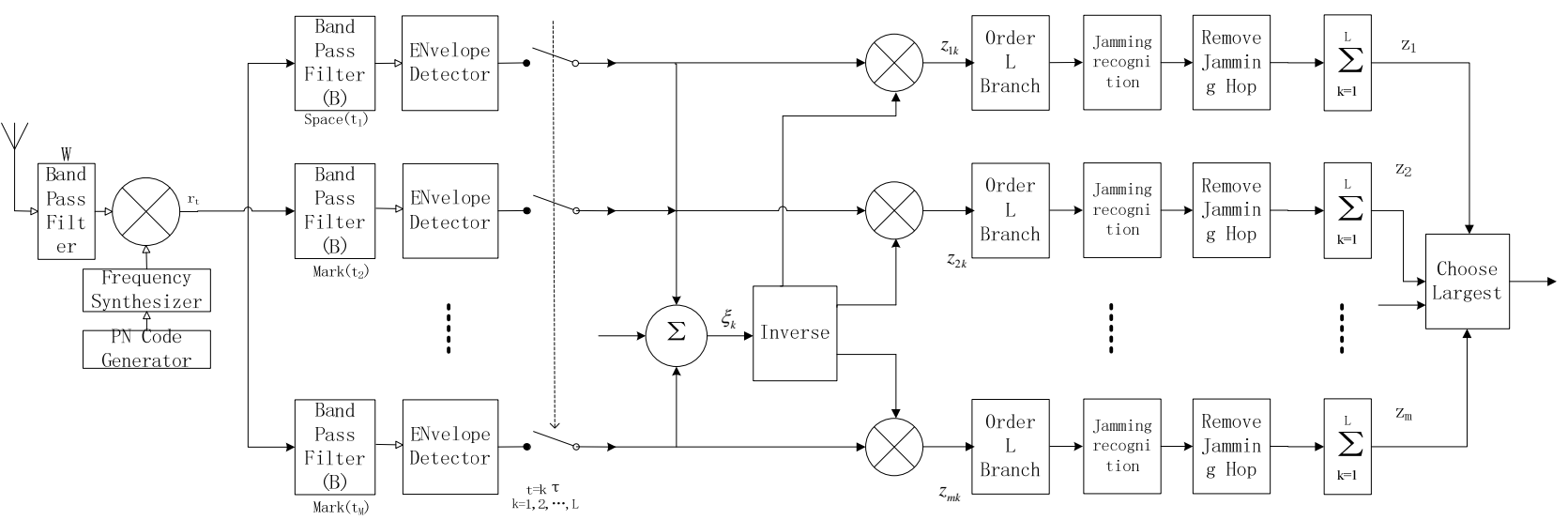

Fig.1. block diagram of JD-SNZ diversity combining receiver technology for L hop/bit MFSK/FFH $\mathrm{m}$ is defined as the number of $\mathrm{M}$ corresponding to the number of points, MFSK that corresponds to the $\mathrm{M}$ frequency point, in the cascade technology, after the jump signal to FFT transform, after the square rate envelope detector, two steps:

The first step: Normalized treatment the envelope of all the frequency point of the normalization process, the current $M$ frequency point envelope value of the cumulative sum of the reciprocal, and then respectively, and the envelope of each frequency point multiplication;

The second step: interference cancellation. L diversity branches from small to large to under each frequency sorted intermediate extraction L diversity branch value, then L diversity sampling values were compared with the median value, if more than the median value of $\mathrm{P}$ times, the diversity branch is disturbed, the diversity of branches of the signal is reduced by half; at last the cumulative envelope value of each frequency point all L diversity branches, and finally the MFSK decision. The jamming deletion of self normalized diversity combining technology, which makes the impact of interference on the system reduced, and effective anti partial band jamming.

First, the envelope value is normalized,

$$
\hat{\mathrm{Z}}_{m l_{i}}=\frac{\mathrm{Z}_{m l_{i}}}{\sum_{m=1}^{M} Z_{m l_{i}}} \quad m=2, \cdots, M
$$

Secondly, the envelope value of L diversity hopping signal at each frequency point is ordered:

$z_{m l_{1}} \leq z_{m l_{2}} \leq z_{m l \frac{L}{2}} \cdots \leq z_{m l_{L-1}} \leq z_{m l_{L}} \quad m=2, \cdots, M$,

$Z_{m l_{1}}$ is the smallest envelope of the $L$ diversity envelope of the first $m$ frequency point, and $z_{m l_{L}}$ is the maximum envelope of the $L$ diversity envelope of the first $m$ frequency point, and $z_{m l \frac{L}{2}}$ is the intermediate envelope value of the $L$ diversity envelope of the first $m$ frequency point.

If the $Z_{m l_{i}}$ is greater than the $Z_{m l \frac{L}{2}}$ envelope P times, the $Z_{m l_{i}}$ is considered to be interference, so that the envelope value of $z_{m l_{i}}=\frac{1}{2} z_{m l_{i}}$.

Finally, the sum of the normalized branch $\mathrm{L}$ is obtained, and the decision vector is obtained:

$Z_{m}=\sum_{i=1}^{L} \hat{z}_{m l_{i}} \quad m=2, \cdots, M$.

Decision $Z_{m}$ maximum value. 


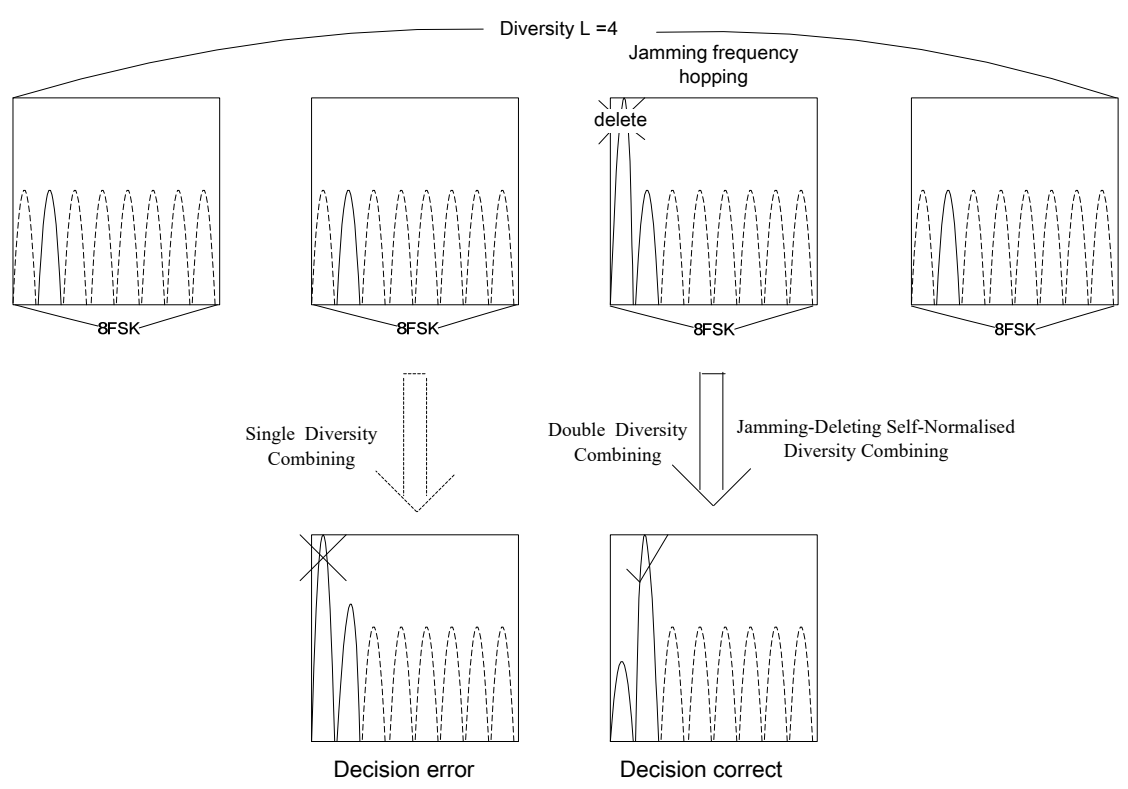

Fig.2. diversity combining technology of $8 \mathrm{FSK} / 4$ diversity

Figure 2 is that the schematic diagram of a simple JD-SNZ algorithm. This scheme adopts the self interference delete cascade normalized diversity technology through frequency hopping anti-jamming performance, remove the energy into the branch, the diversity combining technology to reduce the impact of interference, unlike the traditional diversity technology is the technology of the cascade envelope jump samples of two weighted processing in order to make full use of the fast frequency hopping frequency diversity characteristics, improve diversity gain and enhance the anti-interference ability of the system.

\section{Analysis of JD-SNZ Diversity Combining Technology Performance}

In this section, the performance of the interference cancellation self normalized diversity combining technology is simulated. The simulation environment is as follows: in the worst part of band interference (in the same total power, adjust the interference bandwidth of fast frequency hopping system performance is the worst, the interference factor in the change), in the process of simulation system is selected for MFSK modulation, using the Monte Calor method for simulation in matlab.

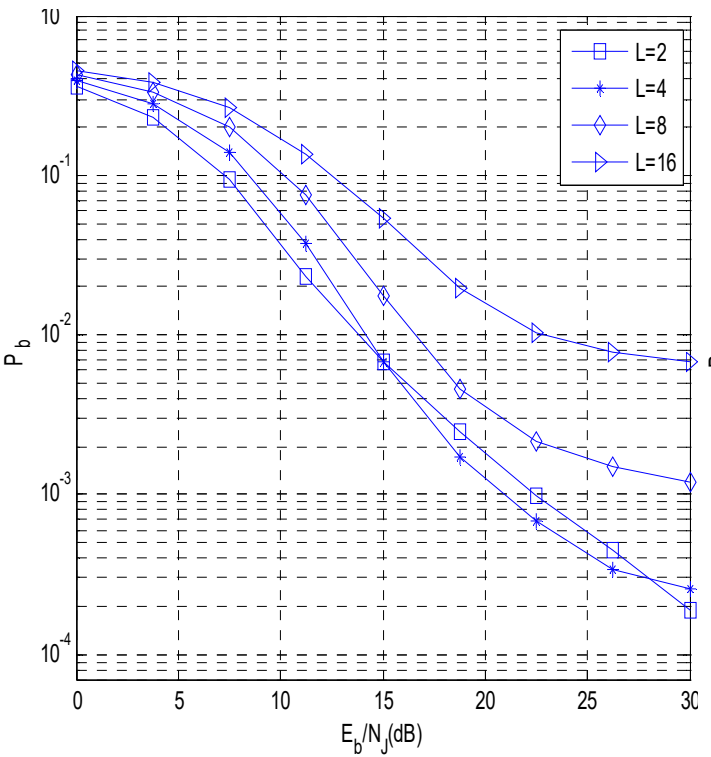

Fig.3. Comparison of different diversity performance for JD-SNZ when $E_{\mathrm{b}} / N_{0}=13.35 \mathrm{~dB}$

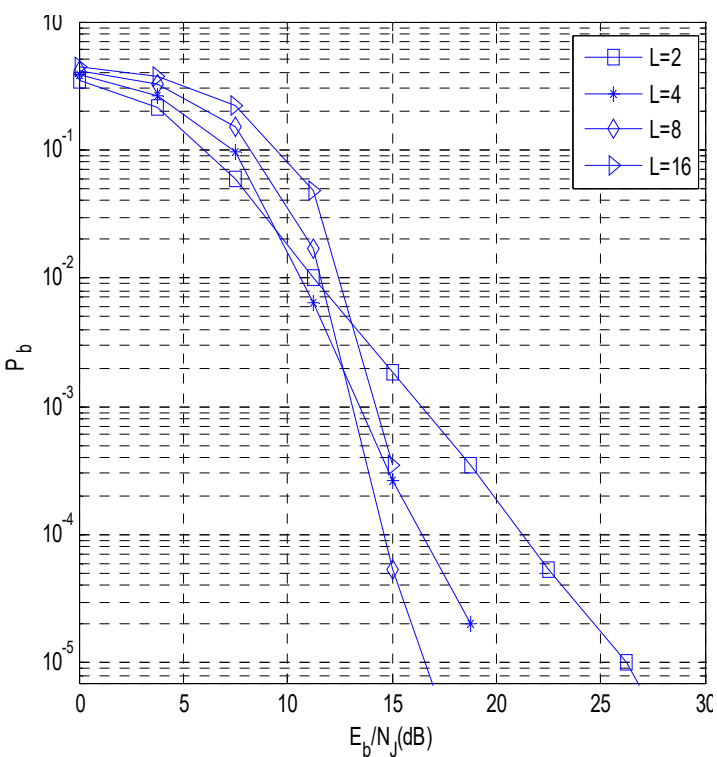

Fig.4. Comparison of different diversity performance for JD-SNZ under no thermal noise 
Figure 3 indicates the performance of the JD-SNZ cascade diversity combining technology with the thermal noise $E_{\mathrm{b}} / N_{0}=13.35 \mathrm{~dB}$ (This signal to noise ratio corresponds to the $1 \mathrm{Hop} / \mathrm{bit}$ of the frequency hopping system without interference in $\mathrm{BER}=10^{-5}$ ) and in the worst partial band jamming. In Figure 3, when the $E_{\mathrm{b}} / N_{\mathrm{J}}<15 \mathrm{~dB}$, diversity number $L=2$ for the best diversity, when the $E_{\mathrm{b}} / N_{J}>15 \mathrm{~dB}$, the diversity number $L=4$ for the best diversity, and its performance is better than the number of 2 .

Figure 4 shows that the performance of the diversity number $\mathrm{L}$ is simulated in the absence of thermal noise (i.e. $N_{0} \rightarrow 0$ ). The results show that with the increase of $L=2,4,8,16$, the performance of anti-jamming is increased, and the diversity effect is obvious. It is indicated that the diversity combining technique can effectively resist partial band interference in the ideal condition of the system (thermal noise). From Figure 4 we see diversity number $L=8$ and $L=16$, the performance curves almost coincide, this phenomenon is because the system performance is improved, and the simulation points is relatively not enough, but it also shows that the thermal noise, the performance increase is not increased with the number of diversity and unlimited increase, when the number reaches a diversity the best value, and then increase the tiller number, the system performance is not improved, the system has the best diversity without a thermal noise number.

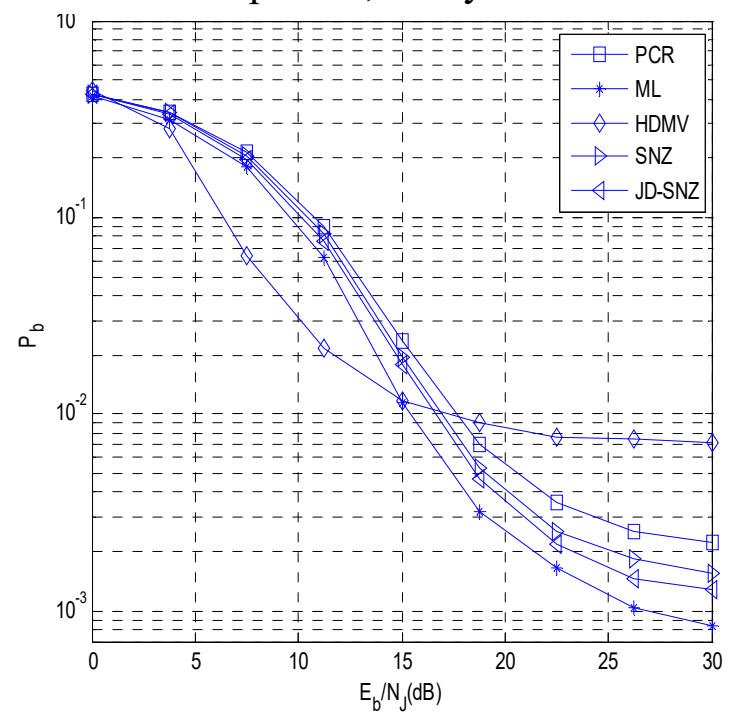

Fig. 5.performance of several diversity combining technology when $\mathrm{L}=8$ under $\mathrm{PBJ}$

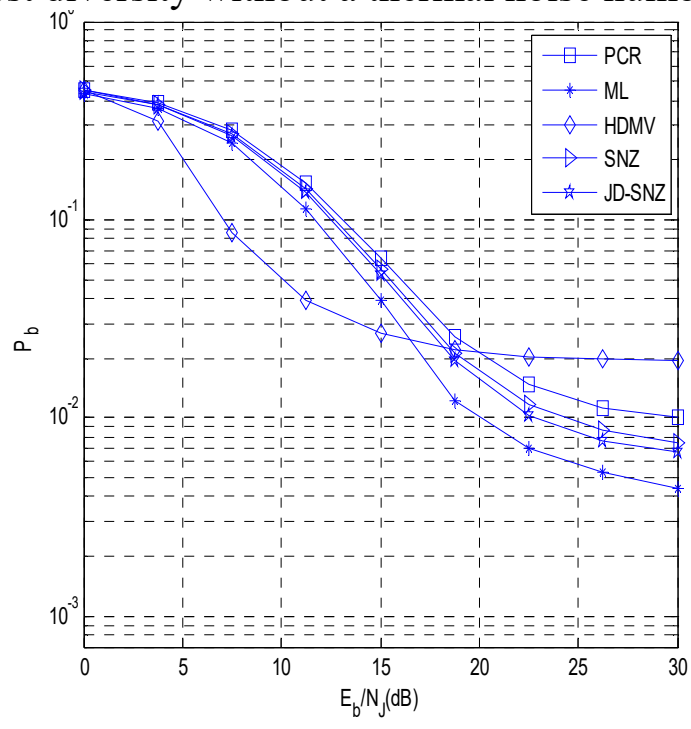

Fig.6. performance of several diversity combining technology when $\mathrm{L}=16$ under $\mathrm{PBJ}$

Figure 5 shows the thermal noise $E_{\mathrm{b}} / N_{0}=13.35 \mathrm{~dB} / L=8$ and the worst partial band interference, Product Combining Receive (PCR),Self-Normalized (SNZ), Hard Decision Majority Vote (HDMV), maximum-likelihood receiver(ML), jamming deleting Self-Normalized (JD-SNZ) Diversity Combining performance comparison. Figure 6 is that the performance comparison of several technologys for diversity $L=16$. When $E_{\mathrm{b}} / N_{\mathrm{J}}<15 \mathrm{~dB}$, the best performance of the hard decision of large number of election technology, when $E_{\mathrm{b}} / N_{\mathrm{J}}>15 \mathrm{~dB}$, the performance from good to bad order is: ML, JD-SNZ, SNZ, PCR, HDMV. The performance of JD-SNZ cascade technology is better than SNZ, but the performance of diversity combining is improved by adding some complexity. JD-SNZ does not need to estimate the noise variance, easy to achieve, ML technology performance is superior, but in the specific implementation, the need to estimate the effective information parameters, to achieve more complex. JD-SNZ in the case of relatively small diversity, the effect is not obvious, and the performance of SNZ.

\section{Conclusion}

Based on the analysis of JD-SNZ Diversity Combining Technology, the time frequency domain diversity technique is a kind of double avoidance technique in the frequency domain and the time 
domain, which has the ability of anti partial band jamming. The performance of SNZ is improved by using the JD-SNZ cascade and anti jamming technology. The implementation complexity is not increased much, the performance has improved, therefore, in the FFH/MFSK system, JD-SNZ technology to improve the performance of the system, it has a certain reference value.

\section{References}

[1]. P.Tardif and E.B. Felstead, Measurement of Diversity-Combining Performance Enhancement for Fast FH. IEEE, 1989. 16-18.

[2] K,T. and Lee.K. Performance analysis of an fft-based ffh/bfsk product-combining receiver with partial-band jamming[J].IEEE Proc.Commun, 1999. Vol.146.No.1: p. 43-48. based

[3] JHONG S. LEE, L.E. MILLER, and Y.K. KIM. Probability of error analyses of a bfsk frequency-hopping system with diversity under partial-band jamming interference-part 111: performance of a square-law self-normalizing soft decision receivers[J].IEEE Trans. Commun, 1986,34(1):669-675.

[4] KAH C T, KOTAND A C, LI K H.Performance study of a maximum-likelihood receiver for FFH/BFSK systems with multitone jamming(ML) [J].IEEE Trans. Commun, 1999,47(5):766-772.

[5] XIAO L, LU J, YAO Y.Diversity combining for FFH system with worst-case partial-band noise jamming[J].IEEE Trans. Commun, 2000,32(5):720-724. 